\title{
An Equilibrium Bid Markup Strategy
}

\author{
${ }^{1}$ Mingxi Wang, ${ }^{2}$ Shulin Liu, ${ }^{3}$ Shouyang Wang, ${ }^{4}$ Luis Coladas Uria \\ ${ }^{*}$ School of International Trade and Economics, University of International Business and \\ Economics, E-mail:mxwang@amss.ac.cn \\ ${ }^{2}$ School of International Trade and Economics, University of International Business and \\ Economics,E-mail:slliu@uibe.edu.cn \\ ${ }^{3}$ Academy of Mathematics and Systems Science, Chinese Academy of Sciences, E-mail: \\ sywang@amss.ac.cn \\ ${ }^{4}$ Department of Statistics and Operations Research, Faculty of Mathematics, Santiago de \\ Compostela Universit, E-mail: luis.coladas@usc.es
}

\begin{abstract}
Setting a bid markup strategy is a very difficult task. Nevertheless, it is important to construction firms or consulting engineering companies because the development of successful bidding strategies is a key factor to their survival in business. Based on the two bidding criteria of the conditional profit ratio and the work force continuity, this short paper first presents the explicit expression of an equilibrium bid markup strategy in procurement auctions. However, the two bidding criteria conflict with each other and tradeoffs must be made. To make tradeoffs between the two bidding criteria, a new bid markup selection making model is then developed by Cobb-Douglas utility function. The model generalizes the classical expected profit model in the sense that the latter's objective function is only a special case of the former. The result shows that the relative importance of a bidding criterion to another has significant effect on the selection of the equilibrium bid markup strategy.
\end{abstract}

Keywords: Bidding Strategy, Procurement, Cobb-Douglas Utility Function

\section{Introduction}

Selecting a successful bid markup or bid price is not only very important to bidders, but also a key factor to the survival of a construction firm or a consulting engineering company. To assist bidders in selection of a most preferred bid markup, Seydel and Olson (1990) present a method in which three bid criteria --- profitability, risk exposure, and work force continuity --- are considered based upon the merging of stochastic bidding models with the analytical hierarchy process (AHP). Later, two new bid criteria, called the conditional positive profit ratio and the expected positive profit ratio, are introduced by Lai et al. (2002). By using the newly introduced criteria, Lai et al. (2002) propose three twocriterion decision models and a single criterion decision model in the determination of the bidder's most preferred markup level ${ }^{1}$. These two papers use discrete numerical examples to demonstrate how to select the most preferred bid markup. However, explicit expression of an equilibrium bid markup strategy is left open in a continuous setting, which motivates the research of this short paper.

Traditionally, a decision objective function with two bid criteria is often analyzed in auction theory. For example, the expected profit decision objective function equals the product of the profit and the winning probability ${ }^{2}$, and the expected utility decision objective function is equal to the utility (or profit's function) multiplied by the winning probability ${ }^{3}$. Thus, these two-criterion decision objective functions are implicitly assumed that bidders have the same preference for the two bid criteria (say, the winning probability and the profit). However, Seydel and Olson (1990) mention that the expected profit maximization may or may not lead to an optimal decision when the decision maker's total utility is considered, depending upon the importance of the profit relative to the other bid criteria. Often bid criteria that are relevant to bidders' decision, such as the profit, the risk reduction, the capital exposure, and the work force continuity, mutually conflict. Typically, as bid markup increases (decreases,

\footnotetext{
${ }^{1}$ Wang et al. (2011) explain the three bidding criteria by demand surplus and supply surplus.

${ }^{2}$ See e.g., McAfee and McMillan (1987), Milgrom (1989), Myerson (1981), Riley and Samuelson (1981), Kim (2007) and Sohangir S. and Seyyedi (2011).

${ }^{3}$ See e.g., Matthews (1983, 1987), Maskin and Riley (1984), Choi et al. (2008) and Chang (2011).
} 
respectively), the work force continuity measured by the winning probability decreases (increases, respectively) gradually while the profit increases (decreases, respectively) gradually. As a result, a change in bid markup strategy leads to inverse changes in the work force continuity and the profit, and tradeoffs must be made. This is another research motivation in this short paper.

To reflect the bidders' reference over each of two bid criteria, or the relative importance of one bid criterion to another, this short paper adopts the Cobb-Douglas utility function to make tradeoffs between the work force continuity and the conditional profit ratio. The reason for this is that, in CobbDouglas utility function $x_{1}{ }^{\alpha} x_{2}^{\beta}$ (where $x_{1}$ and $x_{2}$ are consumption of the two goods), the relative size of $\alpha$ to $\beta$ indicates the relative importance of the goods to consumers. Thus, a new bid markup selection making model is developed, and it generalizes the classical expected profit model in the sense that the latter's objective function is only a special case of the former. The result shows that the relative importance of a bidding criterion to another has significant effect on the selection of the equilibrium bid markup strategy.

This short paper proceeds as follows. Section II introduces the model. Section III derives equilibrium bid markup strategy under the two-criterion decision model proposed by Lai et al. (2002). Based on the Cobb-Douglas utility function, Section IV develops a new decision making model. Section V concludes.

\section{The model}

Consider a case of procurement auctions. The procurer has a single indivisible project contract to procure. The bidder submitting the lowest bid markup gets the project contract.

As in Lai et al. (2002), denote a bid price by $B$, the true cost by $C$ (a random variable), and the estimated cost by $C_{e}$. A bid markup denoted by $M$ is defined as the ratio of the bid price to the estimated cost for the project, i.e., $M=B / C_{e}$. Let $\pi^{-}, \pi^{+}$and $\pi$ be the ratios of the negative profit, the positive profit and the profit to the estimated cost, respectively. $F_{L}($.$) and f_{L}($.$) are the$ probability distribution function and the corresponding density function of the lowest competitive bid markup, respectively.

Given a bid markup $M$, the conditional negative profit ratio in Lai et al. (2002) (called the conditional loss ratio and denoted by E[loss $\mid$ win $]$ in Seydel and Olson (1990)) is

$$
E\left[\pi^{-} \mid \text {win }\right]=\sum_{C>B}\left[\frac{C}{C_{e}}-\frac{B}{C_{e}}\right] P(C),
$$

where $P(C)$ is the probability distribution of the true cost for a given project.

Lai et al. (2002) extend (1) to the case of continuous random variable $X=C / C_{e}$

$$
E\left[\pi^{-} \mid w i n\right]=\int_{M}^{+\infty}(x-M) f(x) d x
$$

and propose a new bid criterion called the conditional positive profit ratio

$$
E\left[\pi^{+} \mid \text {win }\right]=\int_{-\infty}^{M}(M-x) f(x) d x
$$

where $F(x)$ and $f(x)$ are the probability distribution function and the density function of random variable $X=C / C_{e}$, evaluated at $x$, respectively. Then, Lai et al. (2002) prove that the conditional profit ratio, $E[\pi \mid w i n]$, satisfies

$$
E[\pi \mid \text { win }]=E\left[\pi^{+} \mid \text {win }\right]-E\left[\pi^{-} \mid \text {win }\right]=M-E[X]
$$

where $E[X]$ is the expectation value of ratio $X=C / C e$. 
Suppose that bid markup $M$ varies uniformly over some finite interval, Seydel and Olson (1990) demonstrate how to discretize the continuum in order to select a bid markup maximizing the expected profit ratio

$$
E[\pi]=E[\pi \mid \operatorname{win}] P(\operatorname{win}),
$$

where $P($ win $)$ is the probability of winning the project contract. As one numerical example, the example in Seydel and Olson (1990) is used by Lai et al. (2002) to demonstrate and compare their models.

Sequentially, we abstract mathematically the expected profit ratio maximization model in Seydel and Olson (1990) (called a two-criterion decision making model with the conditional profit ratio and the work force continuity in Lai et al. (2002)), and mention its shortage. By (4) and the definition of $F_{L}($.$) , we have$

$$
E[\pi]=(M-E[X])\left(1-F_{L}(M)\right),
$$

And

$$
\frac{\partial^{2} E[\pi]}{\partial M^{2}}=-2 f_{L}(M)-(M-E[X]) f_{L}^{\prime}(M) .
$$

For the special case that $F_{L}($.$) is a uniform distribution function, we have$

$$
\frac{\partial^{2} E[\pi]}{\partial M^{2}}=-2<0,
$$

i.e., $E[\pi]$ is concave in $M$. In other words, Seydel and Olson (1990) and Lai et al. (2002) can certainly select the most preferred bid markup in model (5) under the assumption that the bid markup varies uniformly over some finite interval. In the sense, although Seydel and Olson (1990) and Lai et al. (2002) obtain some interesting results, it is unclear whether or not those results hold for a general probability distribution function.

Practically, estimated cost $C_{e}$ is a constant, and true cost $C$ is a random variable because of future factor price's uncertainty, and bid price $B$ is a decision variable depending on $C$. As $C$ increases, $B$ also increases to make a profit, i.e., $M=B / C_{e}$ is increasing in $X=C / C_{e}$. Therefore, this short paper assumes that decision variable $M$ is the increasing function of ratio $X$, which is different from that in both Seydel and Olson (1990) and Lai et al. (2002).

Suppose that the procurer faces $n$ bidders. For each bidder $i=1,2, \ldots, n$, bidder $i$ values the ratio of the true cost to the estimated cost at an amount $X_{i}$, known only to him. Because the other bidders don't know about this value, it appears to them to be a random variable. It is assumed that the bidders' ratios of the true cost to the estimated cost are independent across all bidders, and that the ratios are drawn from the same distribution function $F(x)$. That is, each bidder agrees that the prior probability that $X_{i}$ is less than $x$ is given by $F(x) . F(x)$ has a positive, continuously differentiable density function $f(x)^{4}$.

As in Seydel and Olson (1990), a bidding company can examine its historical data for the same kind of project, and then roughly know that the true cost is in a finite interval. Let us denote the interval by $[a, b]$ satisfying $F(a)=0$ and $F(b)=1$.

In this short paper, we focus on the case of continuous random variable $X$ with general probability distribution function $F(x)$, and present a Bayes-Nash equilibrium bid markup strategy $M(X)$ to bidders. Because all bidders' ratios $X$ are drawn from the common probability distribution, it is natural to study the symmetric Bayes-Nash equilibrium bid markup strategy.

\footnotetext{
${ }^{4}$ The positive feature of density function $f(x)$ plays an important role in deducing the equilibrium bid markup strategy.
} 
$\mathrm{M}($.$) is called a symmetric Bayes-Nash equilibrium bid markup strategy, if for each bidder i$ with $X_{i}$, bid markup $M\left(X_{i}\right)$ is a best response to the other bidders' bid markups $\left\{M\left(X_{j}\right)\right\}_{j \neq i}$.

\section{Equilibrium bid markup strategy}

When bidders make decision with two criteria --- the conditional profit ratio, $E[\pi \mid$ win $]$, and the work force continuity measured by winning probability, $P(\text { win })^{5}$, according to Seydel and Olson (1990), each bidder's objective is to maximize his expected profit ratio $E[\pi]$,

$$
\max \{E[\pi \mid \operatorname{win}] P(\text { win })\}
$$

Suppose that all but bidder $i$ follow the symmetric Bayes-Nash equilibrium bid markup M(.), and that bidder $i$ with $X_{i}$ bids an amount of $M(t)$. Since $M($.$) is the increasing function of X$, then the winning probability of bidder $i$ is

$$
\begin{aligned}
P(\text { win }) & =\operatorname{Prob}(\text { win })\left[M\left(X_{j}\right)>M(t)\right], j \neq i \\
& =\operatorname{Prob}\left(X_{j}>t, j \neq i\right) \\
& =(1-F(t))^{n-1} \\
& =G(t),
\end{aligned}
$$

where $G(x)=(1-F(x))^{n-1}$.

If bidder $i$ wins the project, the profit ratio earned from the project is the difference between the bid markup and the ratio of the true cost to the estimated cost. Thus, we have

$$
E[\pi \mid w i n]=\left(M(t)-X_{i}\right.
$$

Substituting (8) and (9) into (7), we have

$$
\max _{t}\left\{\left(M(t)-X_{i}\right) G(t)\right\}
$$

By differentiating the objective function in (10) with respect to $t$, we yield

$$
M^{\prime}(t) G(t)+\left(M(t)-X_{i}\right) g(t)
$$

where $g(x)=G^{\prime}(x)$. At a symmetric Bayes-Nash equilibrium, $M\left(X_{i}\right)$ is the best reply to the other bidders' bid markups $\left\{M\left(X_{j}\right)\right\}_{j \neq i}$. Thus, (11) equals zero at $t=X_{i}$. Hence, we have the following differential equation:

$$
M^{\prime}\left(X_{i}\right)+\frac{g\left(X_{i}\right)}{G\left(X_{i}\right)} M\left(X_{i}\right)=\frac{g\left(X_{i}\right)}{G\left(X_{i}\right)} X_{i}
$$

In the equilibrium, the bidder with the lowest true cost submits the lowest bid markup and wins the project. Thus, it is not optimal for bidder $i$ to bid an amount $M(b)>b$. Meanwhile, each bid can not be less than the true cost. Therefore, $M(b)=b$. It is easy to check that

\footnotetext{
${ }^{5}$ To ensure continuity and avoid layoffs, bidding firms must do what is necessary to increase the probability of winning for each project; as firms lower their markup, this probability, and the assurance of work continuity, increase; in this manner, bid acceptance probabilities serve as indicators of continuity (Seydel and Olson, 1990).
} 


$$
M\left(X_{i}\right)=X_{i}+\int_{X_{i}}^{b} \frac{G(y)}{G\left(X_{i}\right)} d y
$$

is a solution of differential equation (12) satisfying the boundary condition of $M(b)=b$.

(13) is merely a necessary condition for $M\left(X_{i}\right)$ to maximize $E[\pi]$, but we claim that it is also sufficient, given that the other bidders follow $M($.$) . In fact, by (10) we have$

$$
\left.E[\pi])\left.\right|_{M=M\left(X_{i}\right)}=\int_{X_{i}}^{b} G(y) d y \text { and } E[\pi]\right)\left.\right|_{M=M(t)}=\left(t-X_{i}\right) G(t)+\int_{t}^{b} G(y) d y .
$$

Noting that $G(x)=(1-F(x))^{n-1}$, then we have

$$
\left.E[\pi])\left.\right|_{M=M\left(X_{i}\right)}-E[\pi]\right)\left.\right|_{M=M(t)}=\int_{X_{i}}^{t}(G(y)-G(t)) d y>0,
$$

regardless of $t<X_{i}$ or $t>X_{i}$. Hence, (13) is the sufficient condition for $M\left(X_{i}\right)$ to maximize $E[\pi]$.

Noting that the bidders' true cost is symmetric (i.e., the bidders' true cost has the identical distribution), we have the following proposition.

Proposition 1 In the procurement auction, when bidders make decision based on model (7), a symmetric Bayes-Nash equilibrium bid markup strategy is given by

$$
M(X)=X+\int_{X}^{b}\left(\frac{1-F(f)}{1-F(X)}\right)^{n-1} d y
$$

By (14),

$$
M^{\prime}(X)=(n-1) \frac{f(X)}{1-F(X)} \int_{X}^{b}\left(\frac{1-F(y)}{1-F(X)}\right)^{n-1} d y>0,
$$

i.e., $M(X)$ is actually increasing in ratio $X$, which is in accordance with practice.

Then, we can make a comparison between the result in Proposition 1 and the correspondingly discrete result in Seydel and Olson (1990). Table 1 is taken from the first column and the fourth column of Table 2 in Seydel and Olson (1990).

Table 1. Expected Profit Ratios

\begin{tabular}{ccccccccc}
\hline$M(\%)$ & 90 & 95 & 100 & 105 & 110 & 115 & 120 & 125 \\
\hline$E[\pi](\%)$ & -15.31 & -9.38 & -4.69 & -1.25 & 0.94 & $1.88^{*}$ & 1.56 & 0.00 \\
\hline
\end{tabular}

Table 1 shows that the expected profit ratio $E[\pi]$ achieves its maximum at $M=115 \%$, since $E[\pi]$ increases gradually over interval $[90 \%, 115 \%]$ and decreases gradually over interval $[115 \%, 125 \%]$.

It implies from (14), however, that decision variable $M$ depends on random variable $X$. In other words, given a realization of random variable $X$, there is a corresponding bid markup to maximize the bidding objective function in (7), i.e., the optimal bid markup changes with the realization of random variable $X$.

Remark 1 (14) implies that the equilibrium bid markup depends on not only the realization of $X$, but also the distribution $F($.) of $X$. Since $0<[1-F(y)] /[1-F(X)]<1(X<y<b)$, then the degree of "markup" (the amount by which $M$ is more than $X$ ) depends on the number of the bidders and 
approaches to 0 (i.e., $M$ approaches to $X$ ) as the number of the bidders becomes more and more larger. Therefore, competition is unprofitable to bidders, but profitable to the procurer.

\section{New model}

In model (7), as the bid markup increases, the conditional profit ratio increases, while the work force continuity decreases. The two criteria of earning the conditional profit ratio (by setting the bid markup high enough) and making work force continuity (by setting the bid markup low enough) conflict with each other, and tradeoffs must be made.

To solve the above tradeoff problem, we evaluate the bidder's equilibrium bid markup by the following maximization problem:

$$
\max _{t}\left\{(M(t)-X)^{\alpha}(G(t))^{\beta}\right\}, \alpha>0, \beta>0, \alpha+\beta=1
$$

where $\alpha$ and $\beta$ are the weights of the conditional profit ratio and the work force continuity, respectively. The role of weights $\alpha$ and $\beta$ serves to express the importance of the conditional profit ratio relative to the work force continuity. Weights $\alpha$ and $\beta$ can also provide useful information, since they indicate what bidders are most concerned about. For example, if $\alpha$ is larger than $\beta$, bidders pay more importance to the conditional profit ratio.

The objective function in (15) is also economically meaningful. It takes a form of CobbDouglas utility functions typically used in economic literature on the consumption of two goods. In Cobb-Douglas utility function $x_{1}{ }^{\alpha} x_{2}{ }^{\beta}, x_{1}$ and $x_{2}$ are consumption of the two goods and the relative size of $\alpha$ to $\beta$ indicates the relative importance of the goods to consumers (Liu and Wang, 2010; Wang et al., 2010).

With the similar way of the deduction of Proposition 1, we can obtain the following proposition.

Proposition 2 In the procurement auction, when bidders use model (15) to represent the relative importance of the conditional profit ratio to the work force continuity, a symmetric Bayes-Nash bid markup strategy is given by

$$
M(X)=X+\int_{X}^{b}\left(\frac{1-F(y)}{1-F(X)}\right)^{(n-1) \frac{\beta}{\alpha}} d y
$$

Remark 2 Model (7) is a special case of our model (15) for $\alpha=\beta=1 / 2$, since both of the two objective functions in (7) and (15) are equivalent for $\alpha=\beta=1 / 2$.

Remark 3 The bidders' preference over the bidding criteria has a significant effect on the selection of the bid markup. By (16), the larger $\beta$ implies that bidders set the lower bid markup. Therefore, Proposition 2 is in accordance with intuition: the larger $\beta$ is, the more emphasis bidders place on the winning probability and on getting the project.

\section{Conclusion}

Based on the two bidding criteria of the conditional profit ratio and the work force continuity, this short paper modifies the model in Lai et al. (2002) and presents explicit expression of an equilibrium bid markup strategy in a procurement auction.

In the real-world situation, however, bidders often face multiple bidding criteria which conflict with each other. Therefore, it is indispensable for bidders to weigh the relative importance of one criterion to another in the determination of a most preferred bid markup. In this short paper, Cobb-Douglas utility function is used to make tradeoffs between the conditional profit ratio and the work force continuity, and a new bid markup selection making 
model is developed. Under the new model, the relative importance of a bidding criterion to another has significant effect on the selection of the equilibrium bid markup strategy, which is accordance with our intuition.

Besides, comparisons are made between the result in this short paper and the corresponding one in Seydel and Olson (1990). It is shown that the result in continuous setting is different from the correspondingly discrete one.

\section{Acknowledgements}

This work was supported partly by the National Natural Science Foundation of China (No. 71001097 and 71171052) and China Postdoctoral Science Foundation (No. 201104167).

\section{References}

[1] Chang S.A., "Networked online auctions: agent behavior and strategic gain", International Journal of Information Processing and Management, vol.2, no.2, pp.59-68, 2011.

[2] Choi J.H., Ahn H. and Han I., "Utility-based double auction mechanism using genetic algorithms", Expert Systems with Applications, vol.34, no.1, pp.150-158, 2008.

[3] Kim Y.S., 'Maximizing sellers' welfare in online auction by simulating bidders' proxy bidding agents", Expert Systems with Applications, vol.32,vno.2, pp.289-298, 2007.

[4] Lai K.K., Liu S.L. and Wang S.Y., "Bid markup selection models by use of multiple criteria", IEEE Transactions on Engineering Management, vol.49, no.2, pp.155-160, 2002.

[5] Liu S.L. and Wang M.X., "Sealed-bid auctions based on Cobb-Douglas utility function", Economics Letters, vol.107, no.1, pp.1-3, 2010.

[6] Maskin E. and Riley J.G., "Optimal auctions with risk averse buyers”, Econometrica, vol.52, no.6, pp.1473-1518, 1984.

[7] Matthews S.A., "Selling to risk averse buyers with unobservable tastes", Journal of Economic Theory, vol.30, no.2, pp.370-400, 1983.

[8] Matthews S., "Comparing auctions for risk averse buyers: a buyer's point of view", Econometrica, vol.55, no3, pp.636-646, 1987.

[9] McAfee R.P. and McMillan J., "Auctions and bidding”, Journal of Economic Literature, vol.25, no.2, pp.699-738, 1987.

[10] Milgrom P., “Auctions and bidding: a primer", Journal of Economic Perspectives, vol.2, no.3, pp.3-22, 1989.

[11] Myerson R.B., "Optimal auction design”, Mathematics of Operations Research, vol.6, no.1, pp.5873, 1981.

[12] Riley J.G. and Samuelson W.F., “Optimal auctions”, American Economic Review, vol.71, no.3, pp.381-392, 1981.

[13] Seydel J. and Olson D.L., "Bids considering multiple criteria", Journal of Construction Engineering and Management, vol.116, no.4, pp.609-623, 1990.

[14] Sohangir S. and Seyyedi M.A., "A service binding method using forward auction algorithm", International Journal of Information Processing and Management, vol.2, no.2, pp.1-7, 2011.

[15] Wang M.X., Liu S.L., Wang S.Y and Lai, K.K., "A weighted product method for bidding strategies in multi-attribute auctions", Journal of Systems Science \& Complexity, vol.23, no.1, pp.194-208, 2010.

[16] Wang M.X., Liu S.L. and Wang S.Y, "The simple economics of bid criteria", Applied Economics Letters, vol.18, no.6, pp.591-594, 2011. 